\title{
Development and construction of an automated curing system for CFRP-Slittapes
}

\author{
Dipl.-Ing. Matthias Bock, Dipl.-Ing. Ivo Meier
}

\begin{abstract}
The usage of pre-impregnated carbon fibers (prepregs) in the aviation is an essential part for manufacturing lightweight constructions. One specific kind of prepregs are slittapes. These are about $1 / 4$ " broad unidirectional fiberreinforced plastics, which are used in winding or fiber placement technology. At this time, components are produced by the mentioned practices and subsequently, they are cured in autoclaves. The main problem with the current state of technology is the rather expensive storage of the semi-finished composite-material.

For this reason, the cure-process should be moved to the beginning of the production process which means to cure the slittapes before production. Afterwards, particular layers are bonded together under pressure. Basis of this innovative processing-method is a new generation of fiber placement heads which are able to process the pre-consolidated slittapes. In order to make further investigations to tape laying heads and the bonding method, it is necessary to be able to produce large quantities of pre-cured slittapes of a consistent quality.

The objective of this paper is to develop a construction which is able to unwind the slittape material from the stock, cure it, and finally to wind it again up on a mandrel.
\end{abstract}

\section{INTRODUCTION}

Fiber reinforced plastics (FRP) are getting more important in technical areas and promise as lightweight and functional materials a large innovation potential. Especially in the passenger and goods traffic, FRP offers high potential savings by reducing the total weight. A fundamental aim in aerospace is to reduce fuel consumption with light and innovative structures. Using fiber-reinforced plastics is one research approach to reach this target [1].

The currently available fiber placement plants are not able to cure the material completely at the process when the material is dropped off [AVK07, p.484]. Currently, the fiber material is processed with the technique above cured in a subsequent autoclaving process. A problem that may occur when storing prepregs is the premature hardening of the exothermic reaction resin. A further disadvantage in the processing of prepreg is the expensive storage. The prepreg must be stored until it is processed at constant $-18^{\circ} \mathrm{C}$, so that there are no chemical reactions of the exothermic resin. Furthermore, the resins used are usually used only up to one year. To avoid the problem of the storage and the costintensive autoclave process, a new way is tested. Figure 1 shows the contrast to the usual procedure. The curing process should be preferred to the manufacturing process to fully cure the slittape before processing.

M. Bock is with the Institute of Composite Structures and Adaptive Systems, German Aerospace Center, 38108 Braunschweig, Germany. (Corresponding author to provide phone: +49-(0)531-295-2366; fax: +49(0)531-295-2838; e-mail: Matthias.Bock@dlr.de).

I. Meier is with the Institute of Joining and Welding, Technische Universität Braunschweig, 38106 Braunschweig, Germany.
Individual layers are glued together under pressure. Basis of this processing method is a new generation of fiber placement head, which is able to process pre-consolidated slittapes. To make further studies on laying heads and gluing processes, it is important to be able to produce large amounts of cured slittapes with consistent quality. Currently, there is no automated process to manufacture consolidate material for the tape-laying process.

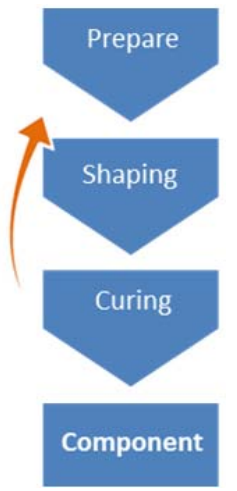

Figure 1. Steps of the manufacturing process with thermoset prepregs

\section{PRE-EXAMINATIONS}

The pre-investigations are analyzing the curing and the heating / cooling behavior. Furthermore the annealing in the partially cured and curved state is examined.

\section{A. Analyzing the curing process by using the DSC}

To gain an insight and to examine a possible acceleration of the curing-process, the slittapes are analyzed by Differential Scanning Calorimetry (DSC). With a rapid curing of slittapes the cycle time can be shorten and the profitability rises. One approach for this is to avoid a long heating up process. Having a relatively small cross-section of $2 \mathrm{~mm}^{2}$ slittapes can rapidly be heated without the danger of local temperature differences. With the so gained research information of temperature, time and cross-linking level, the curing-system can be parameterized later.

DSC is an experimental technique for measuring the energy necessary to establish a nearly zero temperature difference between a test substance (and/or its reaction products) and an inert reference material, while the two samples are subjected to an identical (heating, cooling or constant) temperature program [2].

The measurement of the assays happens by the isothermal principle, i.e. the resin is cured by a constant temperature. A subsequent cooling and heating, highlights potential postcross-linkings. The designated target is to determine the process time to an almost complete cross-linking of the epoxy resin. Figure 2 shows a DSC-thermogram with a curing 
temperature of $180^{\circ} \mathrm{C}$. The specific reaction enthalpy $\Delta \mathrm{h}$ amounts $439 \mathrm{~J} / \mathrm{g}$ and an approximately cross-linking level of $100 \%$ appears at a time of $80 \mathrm{~min}$. The post-cross-linkings are relatively slight and can be neglected.

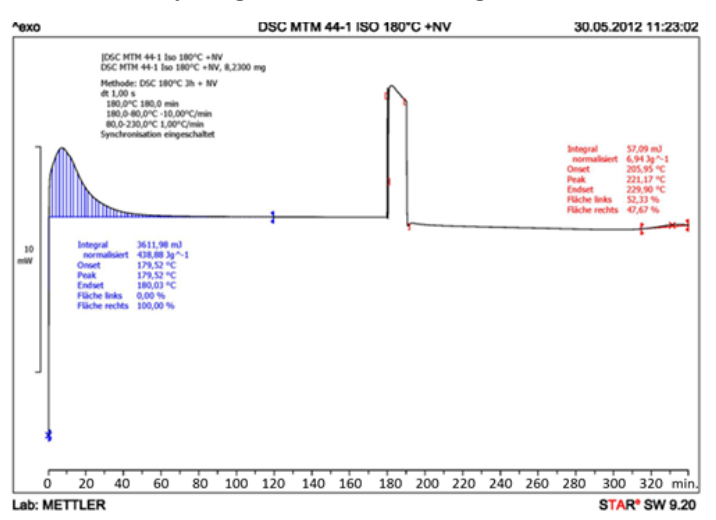

Figure 2. Thermogram of a $180^{\circ} \mathrm{C} \mathrm{DSC}$-analysis

With rising temperature, the duration to an almost complete cross-linking decreases. For designing the curing system, it is important to heat the slittape at a temperature of $180^{\circ} \mathrm{C}$ over a period of at least 80 minutes. Temperatures above $195^{\circ} \mathrm{C}$ should be avoided to prevent resin damages.

\section{B. Analyzing the heating and cooling behavior}

For designing the curing system, an accurate knowledge about the heating and cooling behavior of the fiber composite material is of great importance to parameterize the system with the obtained data.

Regarding the selection of infrared heaters, it is important to ensure that there are different types of radiators. They are structured in short-wave (IR-A), medium-wave (IR-B) and long-wave (IR-C), whereby the term represents the different penetration depth. For the following investigations a shortwave quartz lamp, manufactured by Hereaus, is used. The reason for the selection of a type-A is the high radiation energy. The emitter is a twin-tube version with a length of $340 \mathrm{~mm}$. A special gold reflector on the surface increases the efficiency of a powerful $1200 \mathrm{~W}$ by one-way reflection. Because carbon fiber is a good heat conductor, it is assumed that there are no big temperature differences within the material thickness of $0.3 \mathrm{~mm}$. To adjust the power of the radiator a controller with a potentiometer (also manufactured by Hereaus) is linked to the emitter. The distance between the tube and the prepreg is adjustable. The beam angle of the tube is about $25^{\circ}$, i.e. the radiation field enlarges with increasing distance.

To yield as much energy as possible in the CFRP slittape and to achieve a high efficiency, it is advisable to keep the distance small. During this investigation it is attempted to keep a constant temperature, in order to enable a complete curing of the slittapes. The required time and temperature have been previously analyzed by using the DSC. Figure 3 shows the respective temperature-time curve for the distances 10,20 and $30 \mathrm{~mm}$. Noticeable is the highly discontinuous curve.

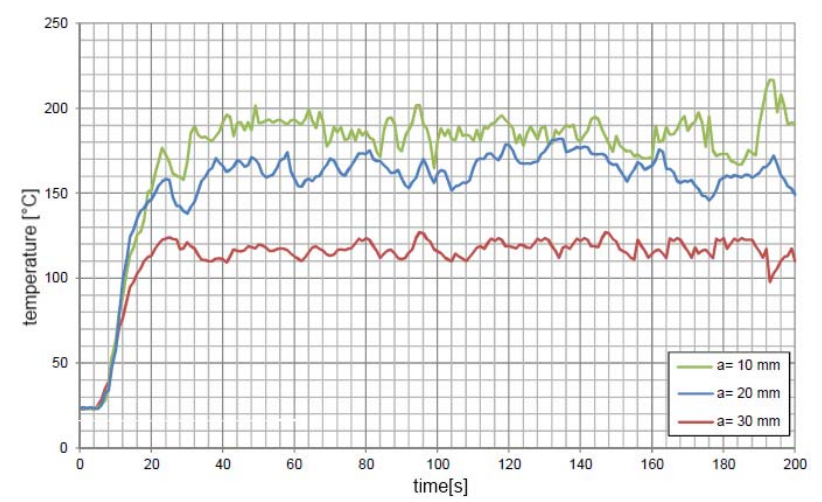

Figure 3. Temperature-time curve for IR-Heating

A reason for this effect could be the influence of air circulation, which is caused by an extraction system in the test room. The slittape material has a thickness of $0.3 \mathrm{~mm}$ and a width of $4 \mathrm{~mm}$. Consequently it has a relatively low heat capacity and thus it is susceptibly for external influences. To verify this assumption, the experimental setup has to be varied. To reduce the influence of air circulation shields are mounted on the bars. Furthermore, these shields have the advantage that they store heat energy and act as a buffer. Figure 4 shows the revised construction.

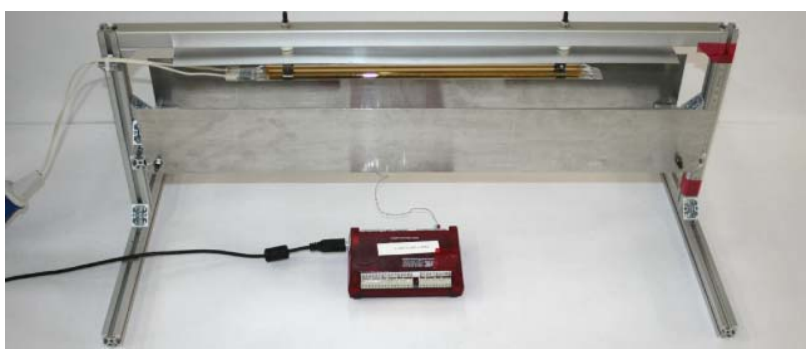

Figure 4. Infrared experiment set-up with additional shields

After a heating up of about 220 seconds, the temperature commutes to a constant level. In the first experimental setup a distance of $20 \mathrm{~mm}$ and a power of $60 \mathrm{~W}$ lead to a slittape temperature of $170^{\circ} \mathrm{C}$. The same parameters lead in this setup (with additional shields) to a plainly higher temperature. After a heating time of 160 seconds the irradiation has to be stopped because the temperature rises over $210^{\circ} \mathrm{C}$. Figure 5 shows the temperature-time curve with additional shields.

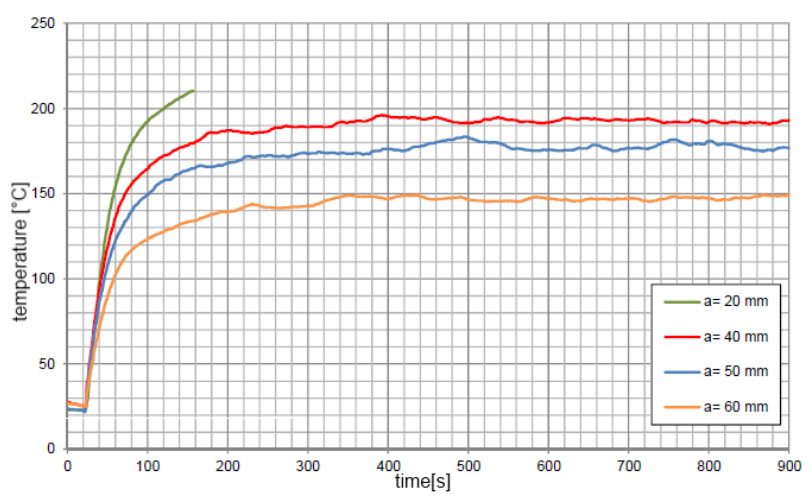

Figure 5. Temperature-time curve for IR-heating with additional shields 
Good compromises for a constant curing-temperature are a distance of $40 \mathrm{~mm}$ and a power of $60 \mathrm{~W}$. A shorter distance would cause a higher efficiency but $60 \mathrm{~W}$ is the technical lower limit of the power amplifier.

\section{Annealing in the partially cured and curved state}

If the cross-linking in an incompletely cured matrix determines the shape of the slittape sufficiently, it would be possible to wind the slittape and subsequently curve it completely. In this case, time and money can be saved with this knowledge and the effectiveness of the device can be increased.

For this study, three slittape specimens are brought in a different cross-linking level $\alpha$ DSC. Analogue to the DSC results, the specimen are partly cured in an oven $\left(180^{\circ} \mathrm{C}\right)$ to $25 \%$ (specimen 1) $50 \%$ (specimen 2) and $75 \%$ (specimen 3 ). Subsequently, the specimens are fixed with adhesive tape on an aluminum tube having a diameter of $70 \mathrm{~mm}$. This corresponds approximately to the roller core diameter, on which the semi-finished product is to be wound. Afterwards the specimens are cured to $\alpha \_$DSC $=100 \%$.

The geometry of the slittapes with fully cross-linked matrix shows that a subsequent annealing has a significant impact to the shape. Depending on the complexity of the precrosslinks, there are different levels of bending radii. Figure 6 shows the test result: the smallest bending radius has specimen 1 , which was cured to a level of $\alpha \_D S C=25 \%$. The bending radius of $40 \mathrm{~mm}$ corresponds approximately to the radius of the aluminum tube. By increasing pre-curing the resultant radius increases. The largest radius $(130 \mathrm{~mm})$ establishes at specimen 3, which has a pre-curing level of $\alpha \_$DSC $=75 \%$.
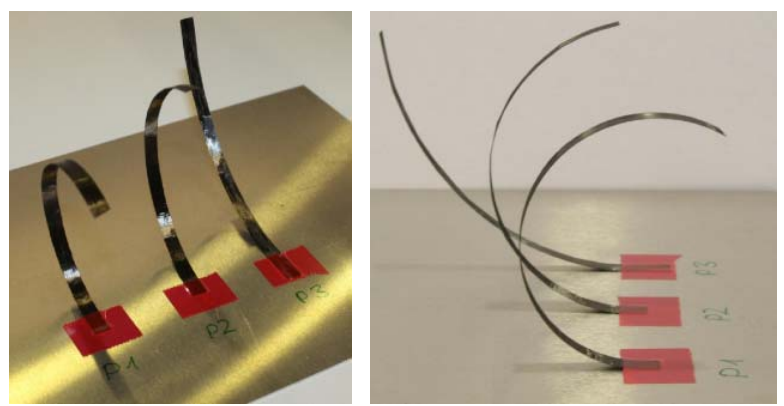

Figure 6. Bending radii of slittapes with different complex pre-crosslinkings.

A subsequent heat treatment in the wound state with partially cured matrix causes a significant curvature of slittapes. Depending on the pre-cross level the curve varies. For the device is it important to cure the slittape completely, to prevent a bending of the material.

\section{Determination of the minimum bend radius in the cured state}

While slittapes are very flexible in the uncured state, the drapability increases with progressive cross-linking of the resin. Within the system, the material has to be deflected by guide elements and finally it has to be wound up on a supply roll. To ensure that there are no material damages during the bending, some bending tests have to be executed. The objective of this study is to identify the minimum bend radius $\mathrm{r}_{\mathrm{B}}$ of the material.

The 4-point bending test consists of a rectangular specimen in a loading fixture that consists of two parallel cylindrical loading pins in contact with one face of the specimen and two more widely spaced cylindrical loading pins in contact with the opposite face. The loading pins are perpendicular and are spaced symmetrically to the center point of the specimen's long axis. The loading geometry has the advantage of generating a uniform, uniaxial stress longitudinally on the tensile side of the bend bar; the size of the uniform tensile region matches the spacing of the narrowly spaced loading cylinders. The large region of uniform tensile stress makes the 4-point bend geometry much preferable to the 3-point bending geometry [3].

Contrary to the expectations, there is no linear relationship between the variables force $F$ and deflection $f$ recognizable. The reason for this phenomenon is the high flexibility of the slittape. There is no fracture enforceable and the specimen is dragged into the test device. The test device is shown in figure 7 . Consequently there are no usable measurements, but this test gives an idea for a possible bending radius as there are no visible material damages. Furthermore, no audible indicators for broken fibers could be perceived. Ultimately, the integrity of the material has to be detected by further studies. The determined bending radius $r_{B}$ is $22.5 \mathrm{~mm}$.

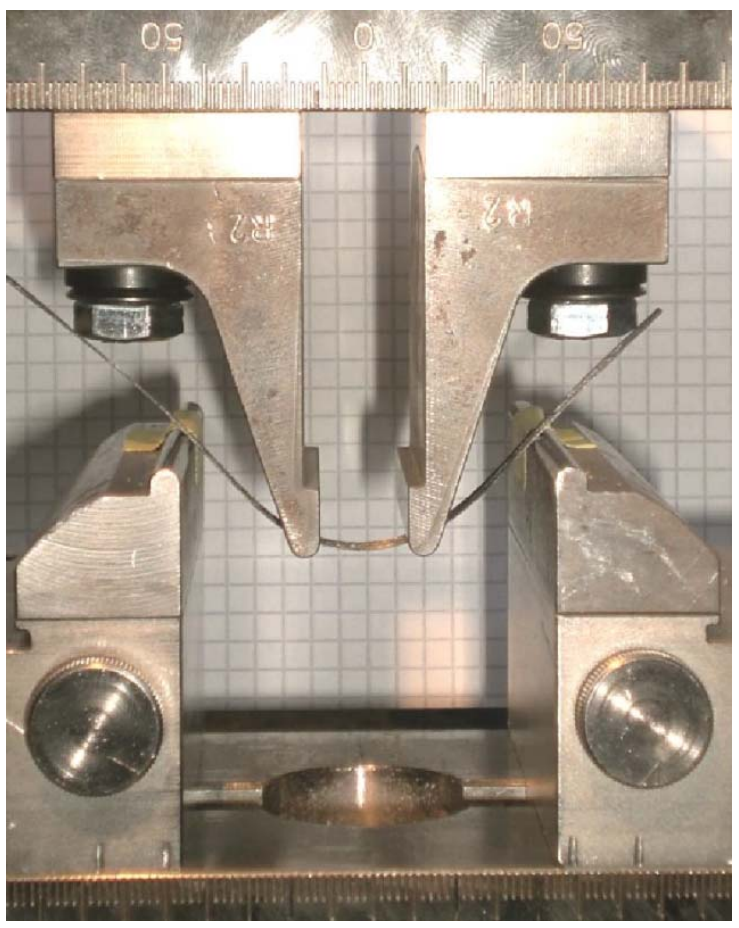

Figure 7. 4-point bending test

To determine the minimum bend radius more exactly an additional bending with only three points is attempted. The advantage of a 3-point bending test is that the span length can be selected lower because the device has only one loading pin. The disadvantage is that the tensile stress is largest at the center of the specimen and drops off immediately as a function of distance away from the center. 
To compare the results with each other, the measurements of five specimens are recorded. The specimens break on average with a deflection of $4.73 \mathrm{~mm}$ and a compression force of $27 \mathrm{~N}$. At approximately $75 \%$ of the maximum deflection an audible damage can be noticed during the exercise. This occurs when fibers tear inside the composite material due to the increasing stress.

The above 3-points approximated maximum bend radius is about $11.5 \mathrm{~mm}$ ( $75 \%$ of the breaking distance). The calculated E-Module is $12.87 \mathrm{kN} / \mathrm{mm}^{2}$.

\section{CONSTRUCTION PART}

An important aspect of the slittape-curing-system is the modular design, which allows to add or leave off particular modules. Additional variants could be a gluing unit, a module for the quality assurance or a cutting unit. Overall, the base version of the prototype should be constructed of three various components: an uncoil-, a curing- and a spoolmodule.

\section{A. Uncoil module}

The aim of the uncoil module is to realize an evenly material uncoiling. The slittape material is stored on a roller core having an inner diameter of $76 \mathrm{~mm}$ and a width of 300 $\mathrm{mm}$. With the help of guide pulleys the horizontal movement is compensated. In addition, the backing film has to be separated from the slittape material and discharged. The uncoil module is shown in figure 8. To prevent a roll-back of roller core and to build a low thread tension, it is necessary to implement a mechanical brake. The seating of the roll is realized by using two cones and one axial force. Two locking levers enable an easy replacement. To avoid slippage between the roll and the cone, there are additional wedges placed in the cone. To ensure an almost frictionless uncoiling, the cones are embedded by using bearings. Due to the axial and radial forces a double-row angular contact ball bearing is used, which can be screwed to a flange with a holding device. The roll socket transfers its torque over a shaft, which is mated with a snug fit to the bearing.

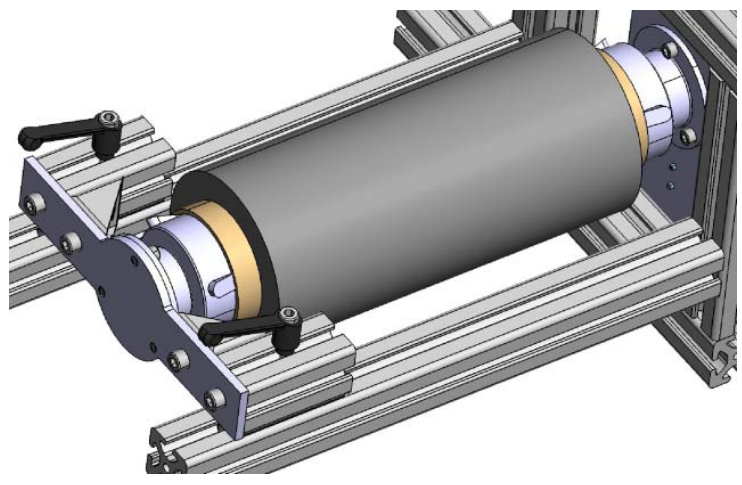

Figure 8. Clamping device for axial bracing

\section{B. Cure module}

The heating process is an essential part of this study. There are basically three mechanisms that cause material warming:

- Conduction: heat transfer within a material

- Convection: carrying of energy by flowing particles

- Thermal radiation: electromagnetic radiation is absorbed
Established heating options are microwaves, contact heating, hot gas and infrared. Important requirements for the selection of a heating method are low cost and the possibility to get easily a long heated area. A short heating up area causes correspondingly a low device speed, because the time of energy input has to be maintained. An induction heating requires a multidirectional fiber alignment and a good electrical contact of the carbon fibers with each other [4]. Because this is not the case, the heating method is excluded. With the help of an efficiency analysis the infrared appears as the preferred variant.

For the construction of the prototype twin-tube emitters, which were previously analyzed, are used. Two emitters are assembled in a row over the material, with a total heating distance of $680 \mathrm{~mm}$. With a curing time of $80 \mathrm{~min}\left(\right.$ at $\left.180^{\circ} \mathrm{C}\right)$ a device speed of $0.51 \mathrm{~m} / \mathrm{s}$ is calculated.

To prevent an adherence between resin and substrate, PTFE bars are used as a horizontal guide. A contactless heating area would be possible at this point but it would require a higher material stress. By using guide pulleys, the slittape is positioned to the PTFE bar. The curing module is shown in figure 9.

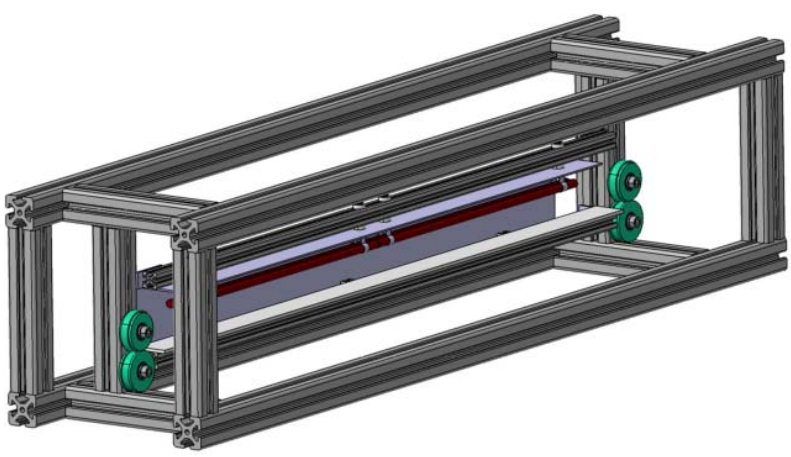

Figure 9. General view of the curing module

\section{Coil module}

The coil module has the function to wind the slittape material iridescent on a roll. Thereby the spool angle has to be variable.

The holding device for the roll is constructed analogue to the uncoil module to reduce common parts and costs. In contrast to the uncoil module, there is a drivetrain instead of a breaking device, which arranges the upwind process and regulates the speed. The drivetrain is a two-phase stepper, which is similar to synchronous motors. They are easy to control and characterized by high durability and reliability.

Another requirement to the curing system is to wind up the slittape iridescently. To solve this task, the use of a linear drive is checked. A linear drive is defined as a device, which moves a carriage transnationally on a rail. In general there are linear axes with three different drive options:

- $\quad$ direct drive using a linear motor

- $\quad$ spindle drive using a stepper motor

- $\quad$ belt drive (also using a stepper motor) 
The advantage of these systems is a high flexibility because the carriage is freely positionable on the rail. Depending on the variant, the feed precision can reach up to $0.02 \mathrm{~mm}$. In addition, a control unit is needed for operation. For the curing system a linear axis with the label LEZ 1 (manufactured by isel) is selected. It is shown with eyelet in figure 10 . With a travel length of $398 \mathrm{~mm}$ and a movement speed of $1.5 \mathrm{~m} / \mathrm{s}$ it satisfies the requirements.

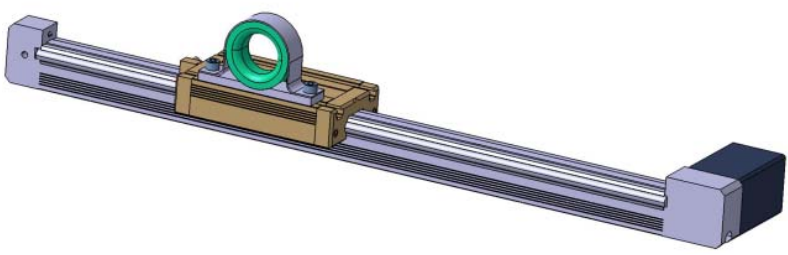

Figure 10. Linear axis for iridescent upwinding

As a guide element an eyelet is used. It is made out of polyethylene, which is also used for the guide rollers. A bent sheet fixes the eyelet to the carriage.

During the installation of the axis, it's important to make sure that the minimum bending radius for the cured Slittape is not deceeded. The deflection in two plains results a superposition of bending and torsion of the CFRP. Accordingly the distances between the deflection points have to be construed. Figure 11 shows the deflection of the Slittape material.

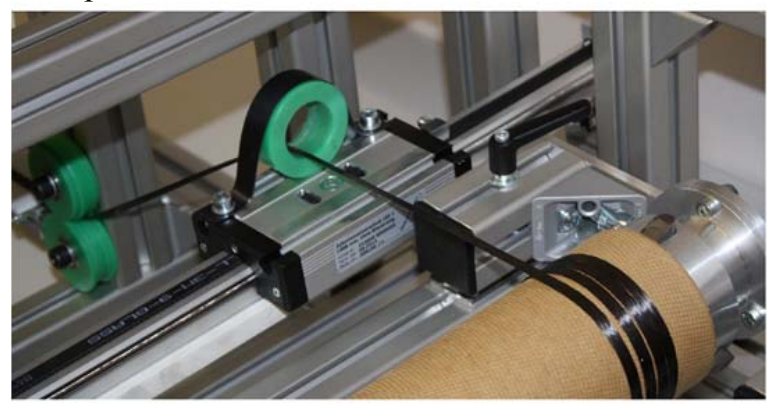

Figure 11. Deflection in the coil module

\section{Controlling the actuators}

The position and speed of the linear axis and the speed of the stepper motor for the drive can be realized by a freely programmable compact controller. The controller integrates the necessary components (ports, power supply, power amplifiers and controls) that are needed to control. The controller operates either permanently connected to a computer or in a "stand-alone" mode. The controller has four outputs. The two unused terminals can be used for controlling of further modules. The advantage is to have a central controller that coordinates the entire actuators.

To make the system work independently, a light barrier is integrated in the device. In case of a completely unwind supply roll, the system recognizes the missing material and stops the system.

\section{Assembly AND TESTING OF THE DEVICE}

After the fabrication of the constructed components, they are assembled to a system. Afterwards, they are examined for their correct function. An important criterion is the torque transfer between the roll and the seating and it is important that there is no slippage. The guideline is a force of $50 \mathrm{~N}$. With a band, the tension is simulated and checked with a spring dynamometer.

The experiment shows that there is even with a traction force of $>100 \mathrm{~N}$ there is no slippage and that the solution with the included feather keys works very well. The deflection pulleys are limited suitability. This leads to an overlay of the desired rotation of the roller and a sliding movement of slittapes. The reason for the inertia is on the one hand the relatively high coefficient of friction of the sealing discs and on the other hand located in the grease of the radial ball bearing. In order to achieve better results, plastic bearings can be used at this point. For setting of the heating power, the lamps are horizontally adjustable at a distance of 0 to 150 $\mathrm{mm}$. The curing module in detail is shown in figure 12 .

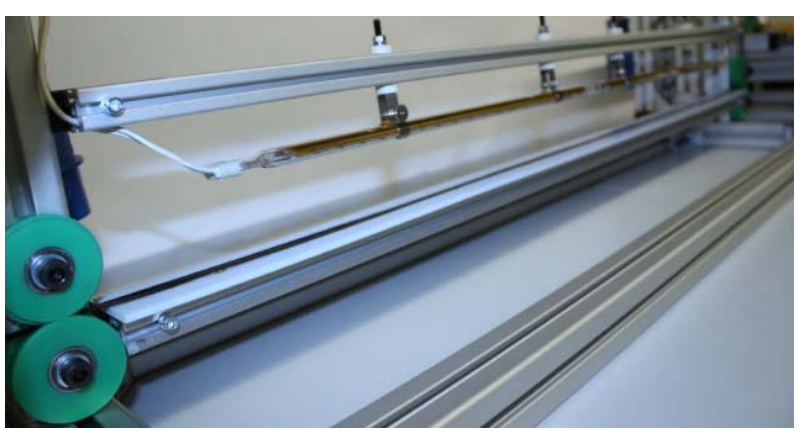

Figure 12. Detail of the curing module

The multi-axis controller is integrated into the device and is easily accessible to the operator. An emergency stop button is located on the front of the controller. The control system sets the position and speed of the linear axis. Moreover, the speed of the stepping motor is regulated. The torque of the drive is transmitted via a toothed belt on the roller holder (Figure 13). In the case of mass production, the timing has to be protected by a shield.

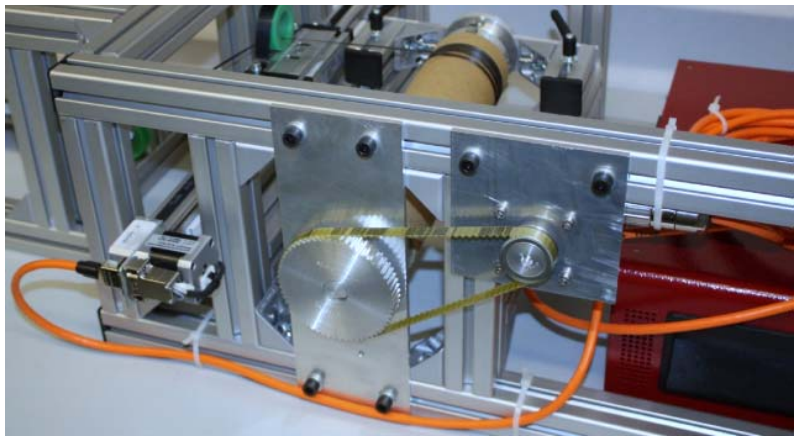

Figure 13. Detail of the roll drivetrain

\section{CONCLUSION}

For testing innovative fiber placement heads, larger amounts of pre-consolidated tapes (cured slittapes) with consistent quality are required. For this purpose an automatic 
curing system is developed. This system uncoils independently the tape and wounds it iridescent on a roll core after a heating process.

For the design and parameterization of the system, it is necessary to conduct a series of preliminary studies. The boundary conditions for a complete cure of slittapes are established by DSC analysis. The matrix of the fiber composite material cures at a temperature of $180^{\circ} \mathrm{C}$ over a period of 80 minutes approximately completely. Lower temperatures result in significantly longer curing times. The glass transition temperature of the resin is approximately $195^{\circ} \mathrm{C}$. With infrared radiation heating, the slittape cures with the system within 220 seconds at a temperature of $180^{\circ} \mathrm{C}$.

To increase the effectiveness of the system, the possibility of subsequent annealing in the wound state is tested. Samples with different curing degrees are prepared and wound on a rotary body, and then fully cured in a tempering furnace. The results are cured samples with different radii of curvature. Prepregs are relatively flexible in the uncured state. The drapability wanes with increasing cross-linking of the matrix. To divert cured tapes, the smallest possible bending radius is determined.

To ensure the greatest variability, the system is built modularly.

On the uncoil module for the supply spool a braking device is added. The force of the tape can be continuously adjusted up to $30 \mathrm{~N}$. Additionally an independent rotation of the coil is prevented. After removal the backing film, the slittape is transferred centrally into the next module.

In the cure module, the slittapes are cured by using the determined boundary conditions such as power and distance via IR emitters. In the coil module the cured slittapes are iridescent wound onto a core with a linear axis with sliding eye. A multi-axis controller contributes the electromechanical components. The whole curing system is shown in figure 14.

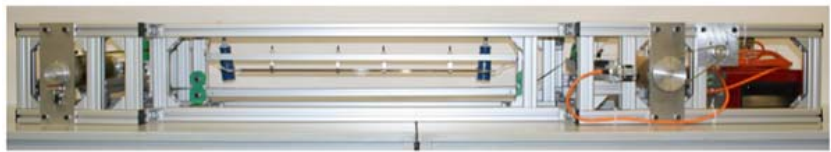

Figure 14. Automated curing system for CFRP- Slittapes

\section{REFERENCES}

[1] M. Perner, C. Krombholz, M. Bock, D. Röstermundt and Dr.-Ing. H. P. Monner, Clarifying Vibrational Issues of an Advanced Automated Fiber Placement Production Plant, Advanced Intelligent Mechatronics, Kaohsiung - Taiwan, 2012

[2] Dr. R. Nicula, Introduction to Differential Scanning Calorimetry, Physics Dept., Rostock University, 2002

[3] Kutz Myer, Handbook of the Material Selection, John Wiley \& Sons, Inc., 2002

[4] Paul Ermanni, Composites Technologies, Institution for mechanical Systems, ETH Zürich, 2007 